\title{
ANALYTIC EXPRESSIONS FOR CONTINUED FRACTIONS OVER A VECTOR SPACE
}

\author{
F. A. ROACH
}

\begin{abstract}
In previous papers, we considered continued fractions obtained from a type of "geometric reciprocal". In this paper we develop certain "analytical" formulas for such continued fractions and, by using these formulas, obtain results analogous to certain classical theorems for ordinary continued fractions.
\end{abstract}

1. Introduction. Throughout this paper, we suppose that $S$ is a real inner product space and that $u$ is a point of $S$ with unit norm. As in [1] and [2], for each point $z$ of $S$, we denote the point $2((z, u)) u-z$ by $\bar{z}$ and the point $\bar{z} /\|z\|^{2}$ by $1 / z$. (We assume that there is adjoined to $S$ a "point at infinity".)

If $S$ is one of $E^{1}, E^{2}, E^{4}$, and $E^{8}$ and $u$ is the point with unit norm and first coordinate 1 , the expression

$$
\frac{1}{1 / x}+\frac{1}{-x}+\frac{1}{(1 / x)-y}
$$

reduces to the product $x y x$ for real numbers, complex numbers, quaternions, and Cayley numbers, respectively. With this in mind, when $y=u$ we denote the value of (1.1) by $x^{2}$. If $a$ and $b$ are points of $S$ and $a$ is not a scalar multiple of $u$, the symbol $a b$ denotes the value of (1.1) when $y=b$ and $x$ is one of the points whose square is $a$. (If $a$ is not a scalar multiple of $u$, there are just two points $x$-one the negative of the other-such that $x^{2}=a$. See (2.1) below.) If $a$ is some scalar times $u$, then $a b$ denotes that scalar times $b$.

Suppose that each one of $a_{1}, a_{2}, a_{3}, \ldots, b_{0}, b_{1}, b_{2}, \ldots$ is a point of $S$. Let $T_{0}(z)$ denote $b_{0}+z$ and, for $n=1,2,3, \ldots$, let $T_{n}(z)$ denote $a_{n}\left(1 /\left(b_{n}+z\right)\right)$. With the convention that $x / y$ denotes $x(1 / y)$,

$$
T_{0} T_{1} T_{2} \cdots T_{n}(z)=b_{0}+\frac{a_{1}}{b_{1}}+\frac{a_{1}}{b_{2}}+\cdots+\frac{a_{n}}{b_{n}+z} .
$$

In this paper, we are concerned with the continued fraction generated by the transformations $T_{0}, T_{1}, T_{2}, \ldots$, i.e., the continued fraction

$$
b_{0}+\frac{a_{1}}{b_{1}}+\frac{a_{2}}{b_{2}}+\frac{a_{3}}{b_{3}}+\cdots
$$

For $z=0$, we refer to the value of (1.2) as the $n$th approximant of (1.3).

If each $a_{n}$ has value $u$, then (1.3) is the type of continued fraction considered in [1]. In [2], the transformations used to obtain the continued

Received by the editors October 24, 1974.

AMS (MOS) subject classifications (1970). Primary 30A22, 40A15.

Key words and phrases. Continued fractions. 
fractions were formed somewhat differently. For a sequence $c_{1}, c_{2}, c_{3}, \ldots$, we defined a transformation $S_{n}$ by

$$
S_{n}(z)= \begin{cases}\frac{1}{c_{n}}+\frac{1}{-c_{n}}+\frac{1}{\left(1 / c_{n}\right)-\left(1 / c_{n}^{2}\right)-z} & \text { if } c_{n} \neq 0, \\ u & \text { if } c_{n}=0 .\end{cases}
$$

Since $-u+1 / S_{n}(z)=-u+c_{n}^{2}\left(1 / c_{n}^{2}+z\right)=c_{n}^{2} z$ (see $\$ 2$ below), $S_{n}(z)$ can be written as $1 /\left(u+c_{n}^{2} z\right)$. If we set $b_{0}=0, a_{1}=u, b_{n}=u, n=1,2,3, \ldots$, and $a_{n+1}=c_{n}^{2}, n=1,2,3, \ldots$, then the $n$th approximant of (1.3) is $S_{1} S_{2} \cdots S_{n}(u)$. Thus (1.3) includes the continued fractions of [2].

2. Properties of the operation. The following Lemma lists the main properties of the operation defined above. We will have occasion to make use of these properties in the last two sections.

LemMa.If each one of $x, y$, and $z$ is a point of $S$, then

(a) $x(y+z)=x y+x z$

(b) $x u=u x=x$;

(c) if $x \neq 0, x(1 / x)=(1 / x) x=u$ and $x[(1 / x) y]=[x(1 / x)] y$;

(d) if $x \neq 0$ and $y \neq 0,1 /(x y)=(1 / x)(1 / y)$;

(e) $\|x y\|=\|x\|\|y\|$; and

(f) if $c$ is a real number, $c(x y)=(c x) y=x(c y)$.

This result is readily established with the aid of the following identity: for every $x$ in $S$ and $y$ in $S$,

$$
x^{2} y=2((x, \bar{y})) x-\|x\|^{2} \bar{y} .
$$

To see that this identity holds true, notice that

$$
\|x+\bar{y}\| x\left\|^{2}\right\|^{2}=\|x\|^{2}\left(1+2((x, \bar{y}))+\|x\|^{2}\|y\|^{2}\right),
$$

and hence $1 /((1 / x)-y)$ is the product of $\left(x-\bar{y}\|x\|^{2}\right)\|x\|^{2}$ times the reciprocal of the right-hand side of this expression. Hence

$$
\begin{aligned}
& \frac{1}{-x}+\frac{1}{(1 / x)-y} \\
& =\frac{\left(1-2((x, \bar{y}))+\|x\|^{2}\|y\|^{2}\right)\left(2((x, y)) \bar{x}-\|x\|^{2}\|y\|^{2} \bar{x}-\|x\|^{2} y\right)}{\|2((x, \bar{y})) x-\| x\left\|^{2}\right\| y\left\|^{2} x-\right\| x\left\|^{2} \bar{y}\right\|^{2}} .
\end{aligned}
$$

By using the properties of the inner product, the denominator of the righthand side of this expression can be written so as to obtain

$$
\frac{1}{-x}+\frac{1}{(1 / x)-y}=\frac{2((x, \bar{y})) \bar{x}-\|x\|^{2}\|y\|^{2} \bar{x}-\|x\|^{2} y}{\|x\|^{4}\|y\|^{2}} .
$$

But $\|2((x, \bar{y})) x-\| x\left\|^{2} \bar{y}\right\|=\|x\|^{2}\|y\|$. Hence the right-hand side of this expression is $1 /\left[2((x, \bar{y})) x-x^{2} \bar{y}\right]-1 / x$. From this, $(2.1)$ follows.

In general, we do not have $(y+z) x=y x+z x, x(y z)=(x y) z$, and $x y=y x$. To see this, we may simply consider appropriate examples in $E^{4}$ with $u=(1,0,0,0)$. In this case, $x y$ may be computed in terms of the 
quaternion product so that examples are easily obtained.

REMARK. As was indicated, in certain cases $x^{2} y$ is a product of the form $x y x$. When $S$ is finite dimensional, the cases mentioned are essentially the only ones. In fact, if we suppose that $S$ is finite dimensional and - is a (not necessarily associative) multiplication on $S$ which is distributive and has the property that if $x$ is in $S, y$ is in $S$, and $c$ is a real number, then $c(x \cdot y)$ $=(c x) \cdot y=x \cdot(c y)$ and if, for every $x$ in $S$ and $y$ in $S, x^{2} y=(x \cdot y) \cdot x$, then $(S, \cdot)$ is a division algebra and hence $S$ is either $E^{1}, E^{2}, E^{4}$, or $E^{8}$.

3. Equivalence transformations and some basic formulas. In this section we give a result which allows us to alter the form of (1.3). We also give some formulas which are closely analogous to the fundamental recurrence formulas for ordinary continued fractions (see [3, pp. 15-20]). At times, the parenthesis indicating a certain association will be omitted with the understanding that the association is from the right to the left. Thus $x y z$ denotes $x(y z), x y z w$ denotes $x(y(z w))$, etc. As before, $x / y$ denotes $x(1 / y)$.

THEOREM 1. Suppose that for $q=1,2,3, \ldots$ and $p=1,2, \ldots, q, c_{p q}$ is a point of $S$ distinct from 0 . Then, for $n=1,2,3, \ldots, T_{0} T_{1} T_{2} \cdots T_{n}(0)$-the nth approximant of (1.3)-is equal to

$$
b_{0}+\frac{a_{1} c_{11}}{c_{11} b_{1}}+\cdots+\frac{c_{n-1, n-1} \cdots c_{1, n-1} a_{n} c_{1, n} \cdots c_{n, n}}{c_{n, n} \cdots c_{1, n} b_{n}} .
$$

By using part (d) of the Lemma, this result may be established by a simple induction argument.

If no $a_{k}$ is 0 , for $q=1,2,3, \ldots, c_{1, q}=1 / a_{q}$, and, for $q=2,3,4 \ldots$ and $p=2,3, \ldots, q, c_{p q}=1 / c_{p-1, q-1}$, then (3.1) is equal to

$$
b_{0}+\frac{1}{b_{1}^{\prime}}+\frac{1}{b_{2}^{\prime}}+\cdots+\frac{1}{b_{n}^{\prime}}
$$

where $b_{k}^{\prime}=c_{k k} \cdots c_{1, k} b_{k}, k=1,2,3, \ldots$ If no $b_{k}=0$, for $q=1,2$, $3, \ldots, c_{q q}=1 / b_{q}$, and for $p \neq q, c_{p q}=u$, then (3.1) is equal to

$$
b_{0}+\frac{a_{1}^{\prime}}{u}+\frac{a_{2}^{\prime}}{u}+\cdots+\frac{a_{n}^{\prime}}{u}
$$

where $a_{1}^{\prime}=a_{1} c_{11}$ and $a_{k}^{\prime}=c_{k-1, k-1} a_{k} c_{k, k}, k=2,3,4, \ldots$ Thus, if no $a_{k}$ is 0 and, for $k=1,2,3, \ldots, b_{2 k}^{\prime}=a_{1}\left(1 / a_{2}\right) \cdots\left(1 / a_{2 k}\right) b_{2 k}$ and $b_{2 k-1}^{\prime}=$ $\left(1 / a_{1}\right) a_{2} \cdots\left(1 / a_{2 k-1}\right) b_{2 k-1},(1.3)$ is equivalent to

$$
b_{0}+\frac{1}{b_{1}^{\prime}}+\frac{1}{b_{2}^{\prime}}+\frac{1}{b_{3}^{\prime}}+\cdots,
$$

while if no $b_{k}$ is 0 and, for $k=1,2,3, \ldots, a_{k+1}^{\prime}=\left(1 / b_{k}\right) a_{k+1}\left(1 / b_{k+1}\right)$ and $a_{1}^{\prime}=a_{1}\left(1 / b_{1}\right)$, then it is equivalent to

$$
b_{0}+\frac{a_{1}^{\prime}}{u}+\frac{a_{2}^{\prime}}{u}+\frac{a_{3}^{\prime}}{u}+\cdots .
$$

Let $A_{1}(z)=a_{1} z, B_{1}=b_{1}$, and, for $n=2,3,4, \ldots$, 


$$
\begin{aligned}
A_{n}(z) & =a_{1}\left(b_{2}+\frac{a_{3}}{b_{3}}+\cdots+\frac{a_{n}}{b_{n}}\right) \cdots\left(b_{n-1}+\frac{a_{n}}{b_{n}}\right) b_{n} z, \\
B_{n} & =b_{n}\left(b_{n-1}+\frac{a_{n}}{b_{n}}\right) \cdots\left(b_{1}+\frac{a_{2}}{b_{2}}+\cdots+\frac{a_{n}}{b_{n}}\right) .
\end{aligned}
$$

Notice that if $S=E^{2}, u=(1,0)$, and $b_{0}=0$, then $A_{n}(u)$ and $B_{n}$ are precisely the $n$th numerator and denominator, respectively, of (1.3). The only reason for defining $A_{n}$ as a function is to insure the correct association; it is always evaluated at either $u$ or at $1 / B_{n}$. With the convention that should the formal expression $0 \cdot \infty$ occur, we will interpret it to be $u$, we have

$$
T_{1} T_{2} \cdots T_{n}(0)=A_{n}\left(1 / B_{n}\right) \text {. }
$$

Thus, the $n$th approximant of $(1.3)$ is $b_{0}+A_{n}\left(1 / B_{n}\right)$.

4. Some applications. The continued fraction (1.3) is said to converge if and only if not infinitely many terms of the sequence $B_{1}, B_{2}, B_{3}, \ldots$ have value 0 and $A_{1}\left(1 / B_{1}\right), A_{2}\left(1 / B_{2}\right), A_{3}\left(1 / B_{3}\right), \ldots$ converges to a finite limit. (Notice that it is possible for some factor in the expression for $B_{n}$ to have value 0 and $B_{n}$ be distinct from 0 . In fact, $0(x+y / 0)$ has value either 0 or $u$ accordingly as $y$ is or is not 0 .)

THEOREM 2. Suppose that for some positive integer $m, a_{m}=0$ while if $m>1$, $a_{n} \neq 0, n=1,2, \ldots, m-1$. Then (1.3) converges if and only if not infinitely many of $B_{1}, B_{2}, B_{3}, \ldots$ are 0 . When convergent, it has value $A_{m-1}\left(1 / B_{m-1}\right)$.

Of course it is necessary that not infinitely many of $B_{1}, B_{2}, B_{3}, \ldots$ have value 0 . Hence, suppose that $N$ is a positive integer such that, for $m>N$, $B_{n} \neq 0$. Notice that $B_{m-1} \neq 0$ for if it were 0 , we would have that $B_{m}=B_{m+1}$ $=B_{m+2}=\cdots=0$. This can be seen in the following manner: Since $a_{m}=0$, then $B_{m}$ is 0 or $b_{m} B_{m-1}$ accordingly as $b_{m}$ is or is not 0 . Thus, if $B_{m-1}$ is 0 , so is $B_{m}$. Likewise, $B_{m+1}$ is 0 or $\left(b_{m}+a_{m+1} / b_{m+1}\right) B_{m-1}$ accordingly as $b_{m}+$ $a_{m+1} / b_{m+1}$ is or is not 0 . Therefore, when $B_{m-1}=0, B_{m+1}=0$. This process may be continued.

Suppose that $n$ is a positive integer larger than both $N$ and $m-1$. Then $B_{n} \neq 0$ and hence

$$
b_{m}+\frac{a_{m+1}}{b_{m+1}}+\cdots+\frac{a_{n}}{b_{n}} \neq 0
$$

Therefore

$$
B_{n}=b_{n}\left(b_{n-1}+\frac{a_{n}}{b_{n}}\right) \cdots\left(b_{m}+\frac{a_{m+1}}{b_{m+1}}+\cdots+\frac{a_{n}}{b_{n}}\right) B_{m-1}
$$

and

$$
A_{n}(z)=A_{m-1}\left(b_{m}+\frac{a_{m+1}}{b_{m+1}}+\cdots+\frac{a_{n}}{b_{n}}\right) \cdots\left(b_{n-1}+\frac{a_{n}}{b_{n}}\right) b_{n} z .
$$

Thus,

$$
A_{n}\left(1 / B_{n}\right)=a_{m-1}\left(1 / B_{m-1}\right)
$$

and hence, (1.3) converges to $A_{m-1}\left(1 / B_{m-1}\right)$. 
The following identity is closely analogous to one for ordinary continued fractions (see [3, pp. 15-16]).

THEOREM 3. If no $a_{k}$ is 0 , then, for $n=1,2,3, \ldots$,

(4.1) $\left\|A_{n}\left(1 / B_{n}\right)-A_{n+1}\left(1 / B_{n+1}\right)\right\|=\left\|a_{1}\right\|\left\|a_{2}\right\| \cdots\left\|a_{n+1}\right\| /\left\|B_{n}\right\|\left\|B_{n+1}\right\|$.

Since no $a_{k}$ is 0 , we may write (1.3) as (3.2). Then according to (2.5) of [1],

$$
\left\|A_{n}\left(1 / B_{n}\right)-A_{n+1}\left(1 / B_{n+1}\right)\right\|=1 /\left(D_{n} D_{n+1}\right)
$$

where, for $k=1,2,3, \ldots$,

$$
D_{k}=\left\|b_{k}^{\prime}\right\|\left\|b_{k-1}^{\prime}+\frac{1}{b_{k}^{\prime}}\right\| \cdots\left\|b_{1}^{\prime}+\frac{1}{b_{2}^{\prime}}+\cdots+\frac{1}{b_{k}^{\prime}}\right\| .
$$

Referring to part (e) of the Lemma, we see that $D_{1}=\left\|1 / a_{1}\right\|\left\|B_{1}\right\|$. Also,

$$
D_{2}=\left\|a_{1}\left(1 / a_{2}\right) b_{2}\right\|\left\|\left(1 / a_{1}\right) b_{1}+1 /\left(a_{1}\left(1 / a_{2}\right) b_{2}\right)\right\|
$$

which, by using parts (a), (d), and (e) of the Lemma, can be reduced to $\left\|1 / a_{2}\right\|\left\|B_{2}\right\|$. By means of induction, we see that for $k=1,2,3, \ldots$,

$$
D_{2 k-1}=\left\|\left(1 / a_{1}\right)\right\|\left\|\left(1 / a_{3}\right)\right\| \cdots\left\|\left(1 / a_{2 k-1}\right)\right\|\left\|B_{2 k-1}\right\|
$$

and

From this, (4.1) follows.

$$
D_{2 k}=\left\|\left(1 / a_{2}\right)\right\|\left\|\left(1 / a_{4}\right)\right\| \cdots\left\|\left(1 / a_{2 k}\right)\right\|\left\|B_{2 k}\right\| .
$$

\section{REFERENCES}

1. F. A. Roach, Continued fractions over an inner product space, Proc. Amer. Math. Soc. 24 (1970), 576-582.

2. The parabola theorem for continued fractions over a vector space, Proc. Amer. Math. Soc. 28 (1971), 137-146. MR 43 \# 762.

3. H. S. Wall, Analytic theory of continued fractions, Van Nostrand, New York, 1948. MR 10, 32.

Department of Mathematics, University of Houston, Houston, Texas 77004 